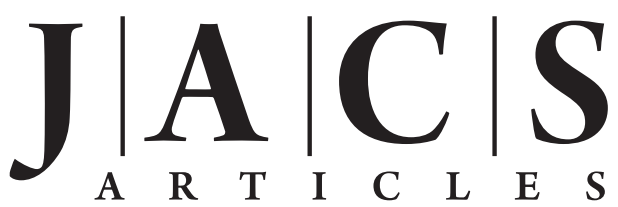

Published on Web 03/10/2010

\title{
Uncovering Compounds by Synergy of Cluster Expansion and High-Throughput Methods
}

\author{
Ohad Levy, ${ }^{\dagger, \ddagger}$ Gus L. W. Hart, ${ }^{\S}$ and Stefano Curtarolo ${ }^{*, \dagger}$ \\ Department of Mechanical Engineering and Materials Science and Department of Physics, Duke \\ University, Durham, North Carolina 27708, Department of Physics, NRCN, \\ P.O. Box 9001, Beer-Sheva, Israel, and Department of Physics and Astronomy, Brigham Young \\ University, Provo, Utah 84602
}

Received December 22, 2009; E-mail: stefano@duke.edu

\begin{abstract}
Predicting from first-principles calculations whether mixed metallic elements phase-separate or form ordered structures is a major challenge of current materials research. It can be partially addressed in cases where experiments suggest the underlying lattice is conserved, using cluster expansion (CE) and a variety of exhaustive evaluation or genetic search algorithms. Evolutionary algorithms have been recently introduced to search for stable off-lattice structures at fixed mixture compositions. The general off-lattice problem is still unsolved. We present an integrated approach of CE and high-throughput ab initio calculations (HT) applicable to the full range of compositions in binary systems where the constituent elements or the intermediate ordered structures have different lattice types. The HT method replaces the search algorithms by direct calculation of a moderate number of naturally occurring prototypes representing all crystal systems and guides CE calculations of derivative structures. This synergy achieves the precision of the CE and the guiding strengths of the HT. Its application to poorly characterized binary Hf systems, believed to be phaseseparating, defines three classes of alloys where CE and HT complement each other to uncover new ordered structures.
\end{abstract}

\section{Introduction}

Predicting the stable structures of alloys from their components is a major challenge of current materials research. ${ }^{1,2}$ Most fundamentally, we should be able to predict whether at thermodynamic equilibrium two elements form ordered structures or phase-separate when mixed. Several well-known empirical and semiempirical methods have been devised for determining phase stability and compound structures in binary metallic systems. They include the Hume-Rothery rules, ${ }^{3}$ the Miedema formation enthalpy method, ${ }^{4}$ Pettifor maps, ${ }^{5}$ and the Zunger pseudopotential radii maps. ${ }^{6}$ These methods produce results largely consistent with existing experimental data and have helped direct a few successful searches for unobserved compounds. ${ }^{7}$ However, they offer a limited response to the challenge of identifying new compounds.

$\mathrm{Ab}$ initio methods explore the phase stability landscape of alloys by calculating the formation enthalpies of a large number of possible structures, identifying the minima at various component concentrations. A minimum free-energy convex hull

\footnotetext{
$\dagger$ Duke University.

$\because \mathrm{NRCN}$.

\& Brigham Young University

(1) Maddox, J. Nature 1988, 335, 201.

(2) Woodley, S. M.; Catlow, R. Nat. Mater. 2008, 7, 937.

(3) Hume-Rothery, W. The Metallic State; Oxford University Press: Oxford, 1931

(4) de Miedema, A. R.; Chatel, P. F.; de Boer, F. R. Physica B \& $C \mathbf{1 9 8 0}$ $100 B, 1$.

(5) Pettifor, D. G. J. Phys. C: Solid State Phys. 1986, 19, 285.

(6) Zunger, A. Phys. Rev. B 1980, 22, 5839.

(7) Pettifor, D. G. J. Phys. C: Solid State Phys. 2003, 15, V13.
}

is constructed from these minimum energy structures. The two major methods of this type are cluster expansion $(\mathrm{CE})^{8,9}$ and high-throughput ab initio calculations (HT). ${ }^{10-12}$ The CE explores structures on specific types of lattices, while the HT explores experimentally known structures representing all crystal systems. The CE allows fast screening of a large set of possible structures limited to a specific configuration space (i.e., superstructures of a parent lattice). It may find derivative superstructures missed by the HT but would fail where structures of different lattice types are revealed by HT calculations. CE calculations have been used with exhaustive evaluation or genetic search algorithms to determine ground-state structures in fixed-lattice systems. ${ }^{13-15}$ The HT approach is practically limited to a smaller number of structures but can reveal compounds with unsuspected lattice types (e.g., an alloy of two hcp elements having a bcc-derived ground state), while missing some favorable structures of a specific type. Recent HT studies include more than 170 structures $^{12}$ and use correlations to accelerate the search. ${ }^{11}$ HT can also be used when the system is poorly characterized, so there is no established information regarding the underlying lattice for the $\mathrm{CE}$ search. A few recent

(8) Sanchez, J. M.; de Fontaine, D. Phys. Rev. B 1982, 25, 1759.

(9) Sanchez, J. M.; Ducastelle, F.; Gratias, D. Physica A 1984, 128, 334.

(10) Chiang, Y.-M.; Sadoway, D. R.; Aydinol, M. K.; Jang, Y.-I.; Huang, B.; Ceder, G. Nature 1998, 392, 694.

(11) Curtarolo, S.; Morgan, D.; Persson, K.; Rodgers, J.; Ceder, G. Phys. Rev. Lett. 2003, 91, 135503.

(12) Curtarolo, S.; Morgan, D.; Ceder, G. Calphad 2005, 29, 163.

(13) Drautz, R.; Diaz-Ortiz, A.; Fahnle, M.; Dosch, D. Phys. Rev. Lett. 2004, 93, 067202 .

(14) d'Avenzac, M.; Zunger, A. Phys. Rev. B 2008, 78, 064102.

(15) Hart, G. L. W.; Forcade, R. W. Phys. Rev. B 2008, 77, 224115. 


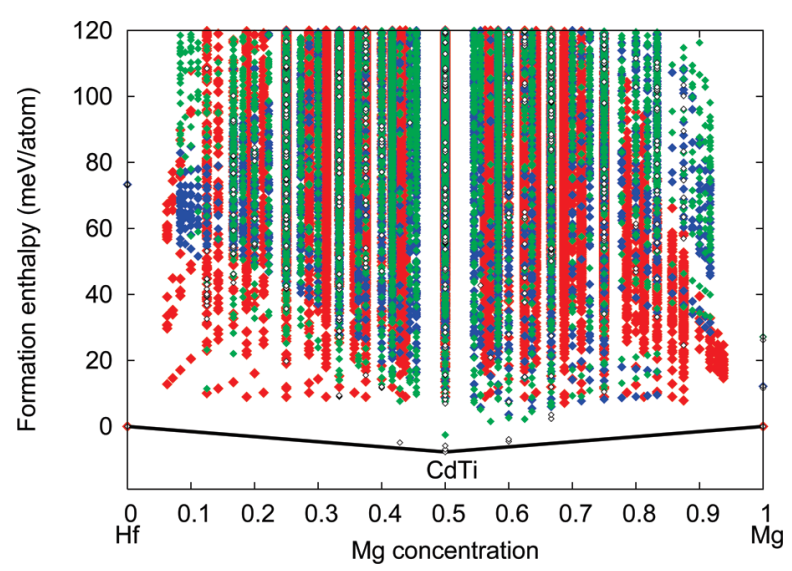

Figure 1. Formation enthalpies of $\mathrm{Hf}-\mathrm{Mg}$ alloys. $\mathrm{HT}$ results are denoted by empty squares and CE results by red, blue, and green markers for hcp-, fcc-, and bcc-derived superstructures, respectively. Formation energies are with respect to the most stable configurations of the pure elements (hcp). Note that all of the hcp superstructures have a positive formation enthalpy.

studies introduced evolutionary algorithms to search for offlattice ordered structures in mixtures with fixed compositions (a priori determined stoichiometries or model interactions). ${ }^{16-19}$ There is currently no satisfactory approach to identifying stable structures of arbitrary composition in binary alloys where the constituent elements or the intermediate ordered structures have different lattice types.

In this paper, we present an integrated approach of $\mathrm{CE}$ and HT calculations that resolves their respective drawbacks. In contrast to the previous methods, where trial structures emerge from simple empirically based guesses or evolutionary algorithms, ${ }^{13-18}$ we use the HT approach to evaluate structures from all crystal systems and guide CE calculations of derivative structures. The evolutionary search is replaced by direct HT calculation of a database of naturally occurring prototypes, taking advantage of the "search" already performed by nature. This approach achieves the precision of the $\mathrm{CE}$ and the guiding strengths of the HT. We demonstrate its effectiveness by finding stable compounds in a few Hf alloy systems experimentally reported to be phase-separating. ${ }^{20,21}$ These results emphasize the complementary strengths of the CE and HT methods and the need for using both in searching for new stable compounds in metallic systems. In addition to the phase stability in the bulk, the integrated approach maps metastable phases (i.e., phases slightly above the convex hull) that may be relevant for the thermodynamic discussion at the nanoscale, where size-dependent chemical potentials ${ }^{22,23}$ are capable of driving a system toward unexpected configurations having different catalytic properties. $^{24,25}$ Such a quantum mechanical description of a variety of metastable natural configurations should be a powerful guide for the rational development of metallic nanocatalysts. ${ }^{26}$

(16) Abraham, N. L.; Probert, M. I. J. Phys. Rev. B 2006, 73, 224104.

(17) Oganov, A. R.; Glass, C. W. J. Chem. Phys. 2006, 124, 244704.

(18) Yao, Y.; Tse, J. S.; Tanaka, K. Phys. Rev. B 2008, 77, 052103.

(19) Filion, L.; Marechal, M.; van Oorschot, B.; Pelt, D.; Smallenburg, F.; Dijkstra, M. Phys. Rev. Lett. 2009, 103, 188302.

(20) Villars, P.; Berndt, M.; Brandenburg, K.; Cenzual, K.; Daams, J.; Hulliger, F.; Massalski, T.; Okamoto, H.; Osaki, K.; Prince, A.; Putz, H.; Iwata, S. J. Alloys Compd. 2004, 367, 293.

(21) Massalski, T. B., Okamoto, H., Subramanian, P. R., Kacprzak, L., Eds. Binary Alloy Phase Diagrams; American Society for Metals: Materials Park, OH, 1990.

(22) Cahn, J. W. Acta Metallurgica 1980, 28, 1333.

(23) Cahn, J. W.; Lärche, F. Acta Metall. 1982, 30, 51. (a)

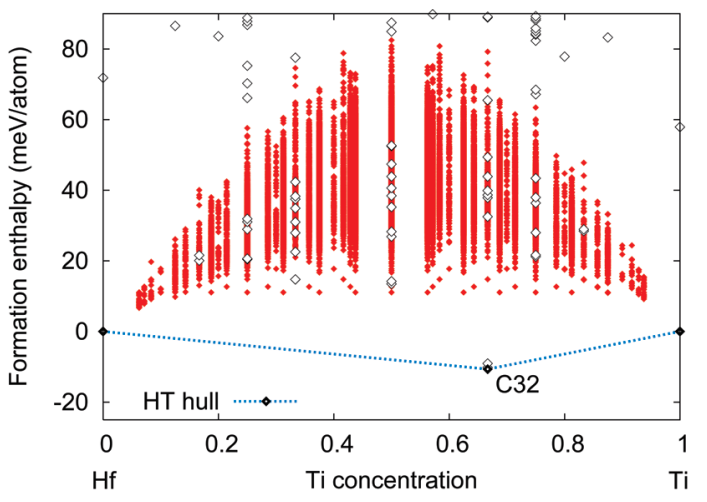

(b)

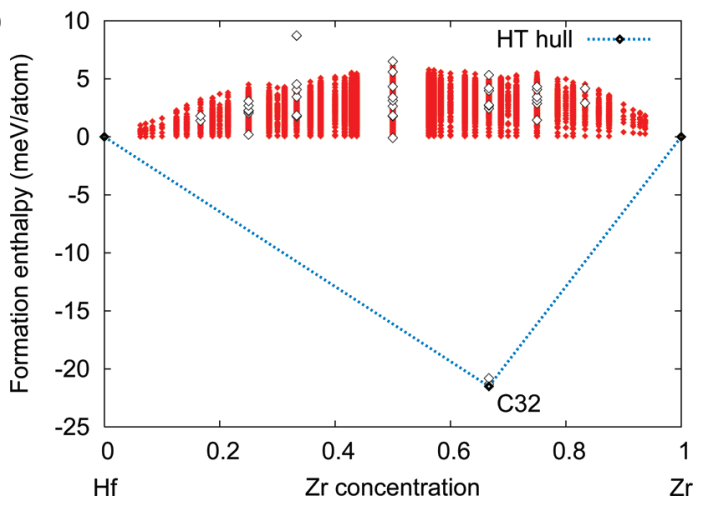

Figure 2. Formation enthalpies of $\mathrm{Hf}-\mathrm{Ti}$ (a) and $\mathrm{Hf}-\mathrm{Zr}$ (b) alloys. The notations are as in Figure 1. The CE finds no structures with negative formation enthalpy. An HT search of 312 structures finds a C32 compound. Most of the HT structures and the CE bcc- and fcc-based structures have high positive enthalpies and are not shown on the plots.

Evolutionary algorithms may be used in conjunction with this approach to improve the efficiency of the CE search (as in refs 14 and 18) or to expand the structure database for the HT search beyond experimentally known structures. However, their practical role in the search for new binary compounds should be expected to be minor because the experimentally available structure database is extensive and has been shown to provide a reliable basis for HT studies of binary systems. ${ }^{12}$ A larger role for evolutionary algorithms may be expected in multicomponent systems where performing such HT studies is a formidable task that has not been tried yet, and the experimentally available database may not represent the entire structure space as comprehensively as in the binary case.

The transition metals of the IIIB and IVB columns of the periodic table are good candidates to demonstrate the advantages of the integrated method. Their binary alloys are all reported as phase-separating, and the available experimental data are very sparse and limited to high temperatures..$^{20,21}$ In particular, the data on Hf binary alloys are available only above 700, 800, and $1000{ }^{\circ} \mathrm{C}$ for $\mathrm{Ti}, \mathrm{Zr}$, and $\mathrm{Sc}$, respectively. ${ }^{20,21} \mathrm{We}$ found no data about the Hf-Y system. All of these elements have an hcp structure, making them perfect for CE investigation. We included the alkaline metal magnesium in this study because it is an hcp element and its binary system with hafnium is also

(24) Harutyunyan, A. R.; Awasthi, N.; Mora, E.; Tokune, T.; Jiang, A.; Setyawan, W.; Bolton, K.; Curtarolo, S. Phys. Rev. Lett. 2008, 100, 195502.

(25) Curtarolo, S.; Awasthi, N.; Setyawan, W.; Jiang, A.; Bolton, K.; Harutyunyan, A. R. Phys. Rev. B 2008, 78, 054105.

(26) Norskov, J. K.; Bligaard, T.; Rossmeisel, J.; Christensen, C. H. Nat. Chem. 2009, 1, 37. 
believed to be phase-separating. ${ }^{20,21}$ These metals have ample technological applications. For example, hafnium and zirconium are used in alloys designed to withstand high temperatures, high pressures, and corrosive environments, which are used in aerospace applications, medical implants and devices, and control and safety mechanisms of nuclear reactors. ${ }^{27}$

\section{Methods}

The HT computations of the low-temperature stability of these five Hf binary systems were carried out using the HT framework AFLOW $^{12,28}$ and ab initio calculations of the energies with the VASP software ${ }^{29}$ with projector augmented wave (PAW) pseudopotentials ${ }^{30}$ and the exchange-correlation functionals parametrized by Perdew, Burke, and Ernzerhof for the generalized gradient approximation. ${ }^{31}$ The energies were calculated at zero temperature and pressure, with spin polarization, and without zero-point motion or lattice vibrations. All crystal structures were fully relaxed (cell volume and shape and the basis atom coordinates inside the cell). Numerical convergence to about $1 \mathrm{meV} /$ atom was ensured by a high-energy cutoff (30\% higher than the maximum cutoff of both potentials) and dense $6000 \mathbf{k}$-point Monkhorst-Pack meshes; 312 structures were considered for every binary system. They included the 176 structures described previously ${ }^{12,32}$ and the prototypes A5, A6, A7, A8, A9, A11, B20, C36, D5 $19, \mathrm{Al}_{2} \mathrm{Zr}_{4}, \mathrm{Al}_{3} \mathrm{Zr}_{2}, \mathrm{Ca}_{7} \mathrm{Ge}, \mathrm{CdTi}$, $\mathrm{Cu}_{3} \mathrm{Ti}_{2}, \mathrm{Ga}_{2} \mathrm{Hf}, \mathrm{Ga}_{4} \mathrm{Ni}, \mathrm{Ga}_{3} \mathrm{Pt}_{5}, \mathrm{Ga}_{4} \mathrm{Ti}_{5}, \mathrm{Hg}_{2} \mathrm{Pt}$, ITl, InTh, LiBMS1/2, ${ }^{33} \mathrm{NbNi}_{8}, \mathrm{NiTi}_{2}, \mathrm{SeTl}, \mathrm{V}_{4} \mathrm{Zn}_{5}$, the complete set of fcc and bcc superstructures with up to five atoms per cell and of hep superstructures up to six atoms per cell.

Cluster expansions were constructed for the four systems indicated to be compound-forming by the HT search. In the initial iteration, the $\mathrm{CE}$ fits were constructed using structures from the HT data, approximately 30 structures for each system and lattice. The CE fits were further refined and verified in an additional iteration, by including first-principles calculations for an additional 5-10 structures identified by a comprehensive enumeration of derivative structures. ${ }^{15}$ Truncation of the cluster expansions was performed using an evolutionary algorithm ${ }^{34,35}$ and a leave-manyout cross-validation scheme, as implemented in the UNCLE code. ${ }^{36}$

\section{Results and Discussion}

The five binary systems studied here were previously reported as phase-separating, but our calculations show that four are actually compound-forming at low temperatures. In three of these systems, $\mathrm{Hf}-\mathrm{Mg}, \mathrm{Hf}-\mathrm{Ti}$, and $\mathrm{Hf}-\mathrm{Zr}$, the new compounds are not hcp-derived structures. The ground state found in the $\mathrm{Hf}-\mathrm{Mg}$ system is CdTi, which is not an hcp-derived superstructure nor a geometrical superstructure of the bcc or fcc lattices (see ref 12). Such a ground state is impossible to find with CE even when expansions of multiple parent lattices are performed, as shown in Figure 1. Another off-lattice ground

(27) Cramer, S. D., Covino, B. S., Eds. ASM Handbook, 10th ed.; ASM International: Metals Park, OH, 2005; Vol. 13B.

(28) Curtarolo, S. Hart, G. L. W. Setyawan, W. Chepulskii, R. V. Levy, O., Morgan, D. AFLOW: software for high-throughput calculation of material properties; http://materials.duke.edu/aflow.html (accessed 2009).

(29) Kresse, G.; Hafner, J. Phys. Rev. B 1993, 47, 558.

(30) Blochl, P. E. Phys. Rev. B 1994, 50, 17953.

(31) Perdew, J. P.; Burke, K.; Ernzerhof, M. Phys. Rev. Lett. 1996, 77, 3865.

(32) Chepulskii, R. V.; Curtarolo, S. Phys. Rev. B 2009, 79, 134203.

(33) Kolmogorov, A. N.; Curtarolo, S. Phys. Rev. 2006, B 73, 180501(R).

(34) Hart, G. L. W.; Blum, V.; Walorski, M. J.; Zunger, A. Nat. Mater. $\mathbf{2 0 0 5}, 4,391$

(35) Blum, V.; Hart, G. L. W.; Walorski, M. J.; Zunger, A. Phys. Rev. B 2005, 72, 165113.

(36) Lerch, D.; Wieckhorst, O.; Hart, G. L. W.; Forcade, R. W.; Müller, S. Model. Simul. Mater. Sci. Eng. 2009, 17, 055003.

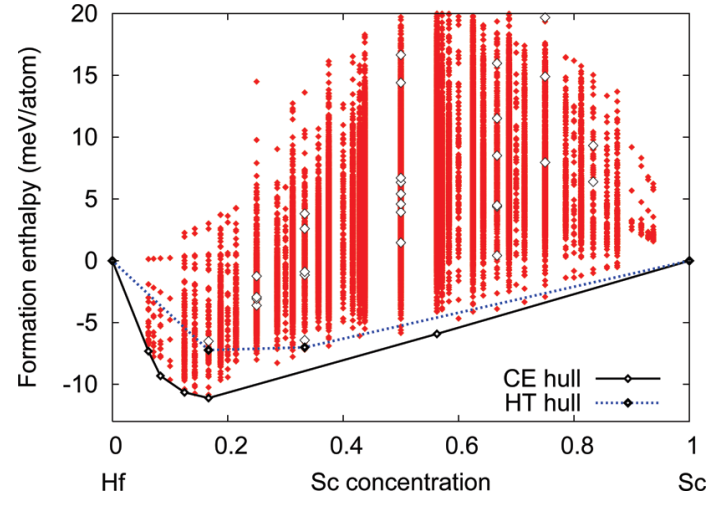

Figure 3. Formation enthalpies of $\mathrm{Hf}-\mathrm{Sc}$ alloys. Both the $\mathrm{HT}$ and $\mathrm{CE}$ searches indicate compound forming. The CE predicts lower-energy structures not included in the HT database.

(a)

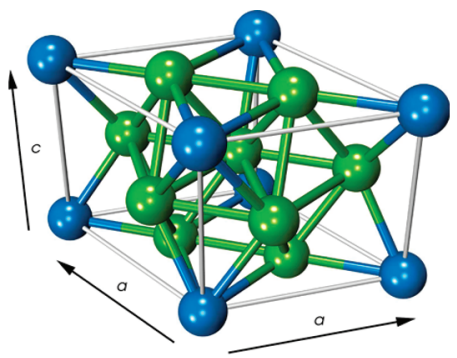

(b)

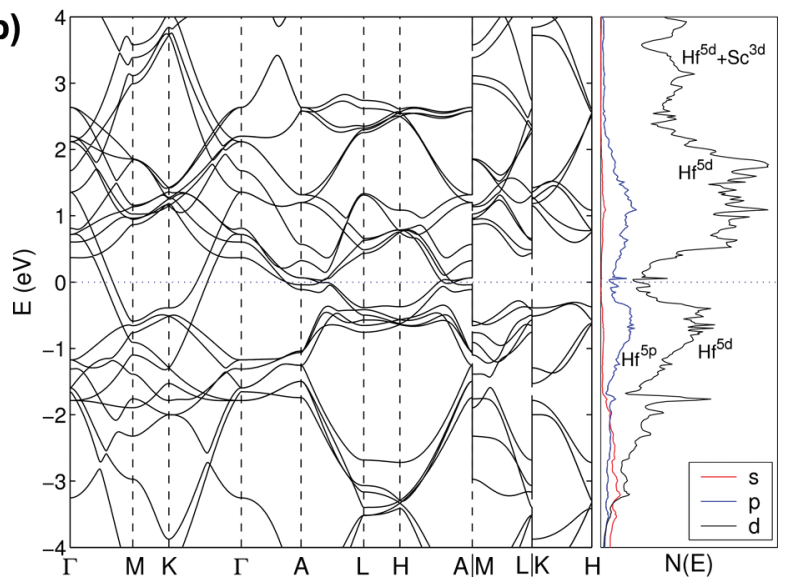

Figure 4. Unit cell of $\mathrm{Hf}_{5} \mathrm{Sc}$ (a), the lowest-energy ground state predicted for the $\mathrm{Hf}-\mathrm{Sc}$ system (Hf, green; Sc, blue) and its band structure (b). This structure has not been observed and does not have a Strukturbericht designation or prototype. The cell dimensions are $a=5.49 \AA, c=5.17 \AA$. All of the bonds marked inside the cell are $3.17 \AA$ long.

state, the $\mathrm{C} 32$ structure (prototype $\mathrm{AlB}_{2}$ ), was found in the $\mathrm{Hf}-\mathrm{Ti}$ and $\mathrm{Hf}-\mathrm{Zr}$ systems. In the $\mathrm{Hf}-\mathrm{Sc}$ system, two hepderived compounds, $\mathrm{Hf}_{5} \mathrm{Sc}$ and $\mathrm{Hf}_{2} \mathrm{Sc}$, were found by the $\mathrm{HT}$ search, and the $\mathrm{CE}$ found several more ground states with lower formation enthalpies. The HT and CE results for these four binary systems are shown in Figures 1-3. The HT formation energies for the various structures are denoted by white squares. The HT convex hulls of minimum energy structures are shown. The breaking points on these hulls are the predicted stable compounds. Figure 1 shows the CdTi ground state found by the HT calculations for the $\mathrm{Hf}-\mathrm{Mg}$ system. The $\mathrm{CE}$ results, denoted by red, green, and blue squares for the hcp-, bcc-, and fcc-derived structures, respectively, mostly lie above the tieline between the pure elements. Although $\mathrm{Hf}$ and $\mathrm{Mg}$ are both hcp elements, some bcc-like superstructures have a lower 
formation enthalpy than the hcp superstructures, all of which have a positive formation enthalpy. Hence, the standard CE approach of considering only the common parent lattice of the pure elements would fail to identify the ordering. The lowest bcc structure, B11 ( $\gamma$-CuTi) at $x=0.5$, was found by both HT and $\mathrm{CE}$ searches.

In Figure 2, it is clearly shown that the CE predicts phase separation in the $\mathrm{Hf}-\mathrm{Ti}$ and $\mathrm{Hf}-\mathrm{Zr}$ systems. All of the structures derived from the common parent lattice of the pure elements (hcp) lie above the elements' tie-line. The bcc- and fcc-derived CE results lie even higher above the line and are not shown. Clearly, the CE method fails in the prediction of the miscibility and determination of the ground state. In Figure 3 , it is shown that the CE convex hull of the $\mathrm{Hf}-\mathrm{Sc}$ system reproduces the compound-forming nature unveiled by the HT results and indicates lower-energy structures. The lowest is the $\mathrm{Hf}_{5} \mathrm{Sc}$ compound shown in Figure 4. Its band structure and density of states indicate an optimum filling of the Hf d-band and a small contribution to the bonding of the Sc d-states. More results about Hf alloys are in ref 37.

\section{Conclusion}

We propose a synergy of the HT and CE methods to identify stable phases of optimal lattice types across the entire composition range of binary alloys. An HT analysis of the system must be performed initially to determine whether the system is miscible or not. Three scenarios arise in cases of miscibility. Scenario I: HT calculations yield new ground states in the original lattice, and the $\mathrm{CE}$ refines them, finding even lower-

(37) Levy, O.; Hart, G. L. W.; Curtarolo, S. Acta Mater. DOI: 10.1016/ j.actamat.2010.01.017. energy structures (represented in this study by the $\mathrm{Hf}-\mathrm{Sc}$ system). Scenario II: HT calculations find an off-lattice ground state and several low-energy structures on a different lattice and guide the $\mathrm{CE}$ to look for additional ground states on other lattices (e.g., the $\mathrm{Hf}-\mathrm{Mg}$ system). Scenario III: HT calculations find an off-lattice ground state that the $\mathrm{CE}$ cannot reproduce $(\mathrm{Hf}-\mathrm{Ti}$ and $\mathrm{Hf}-\mathrm{Zr}$ in this study). This is the most challenging case because the $\mathrm{CE}$ is inapplicable and the search is limited by the completeness of the HT structure library. However, it has been shown that the probability of reproducing the correct ground state with a moderate HT database is very high $\left(\eta^{*} \sim 96.7\right.$, eq 3 in ref 12 ). It should be even higher in the present case due to the larger database. We thus employ the complementary strengths of the CE and HT methods and circumvent their weaknesses. The HT serves as a sampling algorithm of a wide range of naturally selected structure types that can find favorable off-lattice structures and guide the $\mathrm{CE}$ screening to reveal derivative structures. The effectiveness of the integrated approach is demonstrated in this study by identifying new hafnium compounds.

Acknowledgment. We thank Wahyu Setyawan, Mike Mehl, and Leeor Kronik for fruitful discussions, and Michal Jahnátek for help in producing Figure 4. Research was supported by ONR (N00014-07-1-0878, N00014-07-1-1085, N00014-09-1-0921, N0001410-1-0436) and NSF (DMR-0639822, DMR-0650406). We are grateful for extensive use of the Fulton Supercomputer Center at Brigham Young University and Teragrid resources (MCA-07S005). S.C. acknowledges support from the Feinberg Fellowship at the Weizmann Institute of Science.

JA9105623 